\title{
A New Spin on Thermometers for Extremely Low Temperatures
}

\author{
The temperature of an ultracold gas of rubidium atoms is measured precisely using internal \\ quantum states of a single cesium atom.
}

\section{by Stephen R. Clark*}

$\mathrm{T}$ emperature is one of the most widely measured physical quantities. As a notion, it is as old as civilization itself. Yet, scientifically, the meaning and conceptual generality of temperature only fully emerged after intense efforts were made to precisely measure it starting from the 18th century on [1]. That work culminated in the discovery of the absolute temperature scale and revealed the fundamental status temperature has in thermodynamics. Today, temperature measurements, or "thermometry," are pushing this foundation to new extremes by probing smaller energies and smaller length scales, where quantum mechanics plays a dominant role. Advances in such measurements have forced a reassessment of basic thermodynamic quantities [2]. They also hold promise for stimulating novel technologies with so-



Figure 1: A cesium (Cs) atom, shown in blue, immersed in an ultracold gas of rubidium $(\mathrm{Rb})$ atoms acts as a quantum probe of the gas temperature. (APS/Alan Stonebraker)

\footnotetext{
* School of Physics, H. H. Wills Physics Laboratory, University of Bristol, United Kingdom
}

called quantum-enhanced performance [3]. Now, in a new study, Artur Widera from the Technical University of Kaiserslautern, Germany, and colleagues accurately measure the temperature of an ultracold rubidium $(\mathrm{Rb})$ gas using discrete quantized spin states of a cesium (Cs) atom immersed in the gas [4]. This demonstration of quantum-probe thermometry for an ultracold gas promises more accurate measurements of these hard to reach regimes.

Ideally, the temperature of a physical system is measurable without detailed knowledge of the system's inner workings. To achieve that goal, scientists use a probe-another system for which they thoroughly understand the temperature dependence of its physical properties. If the probe is put into contact with the system for a sufficient time, then an energy exchange will occur and cause the probe to equilibrate to the system's temperature. This equilibration allows inference of the system's temperature by the concomitant change in some calibrated property of the probe, such as the column height of a liquid in a capillary tube, the electrical resistance of a conducting element, or the refractive index of a medium.

The frontier of thermometry is thermometer miniaturization with the aim of measuring the difficult-to-access temperatures of very small and cold systems. This goal presents two challenges. First, the probe needs to be much smaller than the system being measured, ensuring that it thermalizes with minimal disturbance to the system. The ultimate minimum size for the probe is a single atom, where information about the system's temperature is mapped onto the atom's quantum state. Second, the characteristic energy scale of the probe needs to be controllable so that it can be tuned to the vicinity of the system's thermal energy, ensuring that the measurement is sensitive. Both these challenges are met by Widera and his co-workers in their new experiment [4].

The team's experimental system consisted of a trapped cloud of just under $10,000 \mathrm{Rb}$ atoms. The atoms were cooled to between 200 and $1000 \mathrm{nK}$, a regime in which the gas behaves like a classical gas. The temperature of such a gas is accurately determinable by time-of-flight measurements by fitting the velocity distribution of atoms in the cloud imaged after the trap is switched off and the cloud is left to expand for some period of time. The system thus serves as a verifi- 


\section{Phys̄īcs}



Figure 2: In the endoergic collisions of interest to the new experiment, the $\mathrm{Rb}$ transitions to a higher-energy state and the $\mathrm{Cs}$ to a lower-energy one. The occurrence of these energy-exchanging transitions depends on the temperature of the $\mathrm{Rb}$ gas. (APS/Alan Stonebraker)

able testbed for an ultracold thermometer.

For the probe, Widera and colleagues turned to the Cs atom, whose internal atomic structure is well characterized (Fig. 1). Cesium possesses seven accessible ground-state hyperfine energy levels for its outer electron, labeled by an angular momentum projection $m_{\mathrm{Cs}}=\{-3,-2,-1,0,1,2,3\}$. Normally these levels all have identical energies. However, applying a weak magnetic field $B$ to the atoms splits the levels into a ladder whose steps have a tuneable energy gap of $\Delta E / 2$. The Cs atoms thus behave like effective quantum-mechanical spins. Rubidium atoms also possess three accessible states, labeled $m_{\mathrm{Rb}}=\{-1,0,1\}$, which turn out to have an energy gap of $\Delta E$ in the same $B$ field.

To use the Cs atoms to determine the Rb cloud's temperature, the team exploited so-called spin-exchange collisions, where quanta of angular momentum are transferred between the $\mathrm{Cs}$ and $\mathrm{Rb}$ atoms. In one type of collision, known as an endoergic collision, the $\mathrm{Rb}$ is pushed into a higherenergy state and the Cs into a lower-energy state (Fig. 2). This process requires $\Delta E / 2$ of additional energy, which is provided by the motion of the $\mathrm{Rb}$ atoms. The occurrence of these collisions depends on the availability of kinetic energy - and therefore the temperature — of the $\mathrm{Rb}$ cloud. The spread in the distribution of the Cs atoms' spin-state populations induced by these collisions thus encodes information about the gas temperature.

The team measured the populations of a handful of Cs atoms after $3 \mathrm{~s}$, by which time the system had reached a steady state. They observed that the steady-state fluctua- tions in the Cs atoms' energies were linearly related to the temperature of the $\mathrm{Rb}$ cloud, as independently determined by time-of-flight measurements. The same relationship was found for different applied magnetic fields, different densities of the $\mathrm{Rb}$ cloud, and different initial states. This robust result thus convincingly demonstrates that thermometry can be performed using a single-atom quantum probe without the need for detailed model fitting. However, the relatively long time required to reach the steady state is not always accessible. To overcome this problem, Widera's team fitted the experimental data describing the evolution of the Cs probe populations before steady state to a specific microscopic rate model. In this way, they could extract the temperature of the system after just $350 \mathrm{~ms}$ of interaction. A theoretical analysis of this approach indicates that only three collisions are needed to obtain a temperature measurement. Furthermore, these measurements are nearly an order of magnitude more sensitive than those performed in the steady state.

This experiment is a fascinating demonstration of a rapid quantum-probe temperature measurement, where the information extracted is maximized and the perturbation to the system is minimized. Future work will undoubtedly exploit the quantumness of the probe beyond spin populations [5] and also utilize universal nonequilibrium properties to avoid the specific model fitting needed here for such measurements [6].

Quantum-probe thermometry has many advantages over conventional time-of-flight measurements, since it is nondestructive, minimally invasive, and spatially localized. The most immediate application is to cold-atom quantum simulations [7], notably strongly interacting fermionic atoms trapped in optical lattices. Such simulation experiments aim to investigate important model systems for which we lack a complete understanding of the physics. This deficiency of knowledge makes it notoriously difficult to directly measure their temperature. Consequently, quantum-probe thermometry will likely be a crucial ingredient for quantum simulations that aim to resolve longstanding questions about these model systems, such as whether they exhibit high-temperature superconductivity [8].

This research is published in Physical Review X.

\section{REFERENCES}

[1] T. J. Quinn and J. P. Compton, "The foundations of thermometry," Rep. Prog. Phys. 38, 151 (1975).

[2] J. Goold et al., "The role of quantum information in thermodynamics - a topical review," J. Phys. A 49, 143001 (2016).

[3] C. L. Degen et al., "Quantum sensing," Rev. Mod. Phys. 89, 035002 (2017).

[4] Q. Bouton et al., "Single-atom quantum probes for ultracold gases boosted by nonequilibrium spin dynamics," Phys. Rev. X 10, 011018 (2020).

[5] M. Mehboudi et al., "Thermometry in the quantum regime: Recent theoretical progress," J. Phys. A 52, 303001 (2019). 
[6] T. H. Johnson et al., "Thermometry of ultracold atoms via nonequilibrium work distributions," Phys. Rev. A 93, 053619 (2016).

[7] T. H. Johnson et al., "What is a quantum simulator?" EPJ Quantum Technol. 1, 1 (2014).
[8] A. Mazurenko et al., "A cold-atom Fermi-Hubbard antiferromagnet," Nature 545, 462 (2017).

10.1103/Physics. 13.7 